\title{
ON BOUNDED WEAK AND STRONG SOLUTIONS OF NON LINEAR DIFFERENTIAL EQUATIONS WITH AND WITHOUT DELAY IN BANACH SPACES
}

\author{
ADEL MAHMOUD GOMAA
}

\begin{abstract}
Assume that $E$ is a Banach space, $B_{r}=\{x \in E:\|x\| \leq r\}$ and $C\left([-d, 0], B_{r}\right)$ is the Banach space of continuous functions from $[-d, 0]$ into $B_{r}$. Consider $f: \mathrm{R}^{+} \times E \rightarrow E ; f^{d}:[0, T] \times$ $C\left([-d, 0], B_{r}\right) \rightarrow E$; for each $t \in[0, T]$ the mapping $\theta_{t} \in C\left([-d, 0], B_{r}\right)$ is defined by $\theta_{t} x(s)=x(t+s), s \in[-d, 0]$ and let $A(t)$ be a linear operator from $E$ into itself. In this paper we give existence theorems for bounded weak and strong solutions of the nonlinear differential equation
\end{abstract}

$$
\dot{x}(t)=A(t) x+f(t, x), \quad t \in \mathrm{R}^{+},
$$

and we prove that, with certain conditions, the differential equation with delay

$$
\dot{x}(t)=L(t) x(t)+f^{d}\left(t, \theta_{t} x\right), \quad \text { if } \quad t \in[0, T]
$$

has at least one weak solution where $L(t)$ is a linear operator from $E$ into $E$. Moreover, under suitable assumptions, the problem $(\mathrm{Q})$ has a solution. Furthermore under a generalization of the compactness assumptions, we show that $(\mathrm{Q})$ has a solution too.

\section{Introduction and preliminaries}

In this paper the dual space of an infinite dimensional Banach space $E$ will be denoted by $E^{*}$ and the pairing between $E$ and $E^{*}$ is denoted by \langle\rangle . Denote by $E_{w}$ the Banach space $E$ endowed with the weak topology. We denote the closed unit sphere in $E$ by $B_{1}=\{x \in E:\|x\| \leq 1\}$. Further, let $\mathscr{L}\left(\mathrm{R}^{+}, E\right)$ be the space of measurable functions $u: \mathrm{R}^{+} \rightarrow E, \mathscr{L}(E)$ be the space of linear operators from $E$ into itself and $\lambda$ be the Lebesgue measure on $I=[0, T]$. Furthermore, let $C(I, E)$ be the space of all continuous functions from $I$ to $E$ with the usual supremum norm and $C_{w}(I, E)$ be the space of all weakly continuous functions from $I$ to $E$ endowed with the topology of weak uniform convergence. Let $C([-d, 0], E)$ be the Banach space of continuous functions from the closed interval $[-d, 0](d \geq 0)$ into $E$ and $\mathscr{B}$ be the family of all bounded subsets of $E$.

Let $\mathscr{M}=\mathscr{M}\left(\mathrm{R}^{+}, E\right)$ be a Banach space of measurable functions $x: \mathrm{R}^{+} \rightarrow$ $E$ with $\|x\| \in \mathscr{M}\left(\mathrm{R}^{+}, \mathrm{R}\right),\|x\|_{\mathscr{M}}=\|\| x\|\|_{\mathscr{M}\left(\mathrm{R}^{+}, \mathrm{R}\right)}$, where 
(1) $\mathscr{M}\left(\mathrm{R}^{+}, \mathrm{R}\right) \subset \mathscr{L}\left(\mathrm{R}^{+}, \mathrm{R}\right)$,

(2) $\mathscr{M}\left(\mathrm{R}^{+}, \mathrm{R}\right)$ contains all essentially bounded functions with compact support,

(3) if $x \in \mathcal{M}\left(\mathrm{R}^{+}, \mathrm{R}\right), y: \mathrm{R}^{+} \rightarrow \mathrm{R}$ is measurable with $|y| \leq|x|$, then $y \in \mathscr{M}\left(\mathrm{R}^{+}, \mathrm{R}\right)$ and $\|y\|_{\mathscr{M}\left(\mathrm{R}^{+}, \mathrm{R}\right)} \leq\|x\|_{\mathscr{M}\left(\mathrm{R}^{+}, \mathrm{R}\right)}$,

(4) if $x \in \mathscr{M}\left(\mathrm{R}^{+}, \mathrm{R}\right), x_{n} \in \mathscr{M}\left(\mathrm{R}^{+}, \mathrm{R}\right),\left|x_{n}\right| \leq|x|$ and $\lim _{n \rightarrow \infty} x_{n}(t)=0$ a.e. on $\mathrm{R}^{+}$, then $\lim _{n \rightarrow \infty}\left\|x_{n}\right\|_{M\left(\mathrm{R}^{+}, \mathrm{R}\right)}=0$.

Let $\mathscr{M}^{\prime}$ denote the associate space to $\mathscr{M}$ [20].

Definition 1.1. The map $\gamma: \mathscr{B} \rightarrow \mathbf{R}^{+}$is called a measure of strong (weak) noncompactness on $\mathscr{B}$ if, for $U, V \in \mathscr{B}$,

$\left(\mathrm{M}_{1}\right) U \subset V \longrightarrow \gamma(U) \leq \gamma(V)$,

$\left(\mathrm{M}_{2}\right) \gamma(U \cup V) \leq \max (\gamma(U), \gamma(V))$,

$\left(\mathrm{M}_{3}\right) \gamma(\overline{\mathrm{conv}} U)=\gamma(U)$,

$\left(\mathrm{M}_{4}\right) \gamma(U+V) \leq \gamma(U)+\gamma(V)$,

$\left(\mathrm{M}_{5}\right) \gamma(c U)=|c| \gamma(U), c \in \mathrm{R}$,

$\left(\mathrm{M}_{6}\right) \gamma(U)=0 \Longleftrightarrow U$ is relatively strongly (weakly) compact in $E$,

$\left(\mathrm{M}_{7}\right) \gamma(U \cup\{x\})=\gamma(U), x \in E$.

Definition 1.2. A function $u:[a, b] \rightarrow E,(a, b) \in \mathrm{R}^{2}$, is called:

(a) Pettis integrable if for any measurable subset $D$ of $[a, b]$ there is an element $v_{D}$ in $E$ such that $\left\langle v_{D}, f\right\rangle=\int_{D}\langle u(s), f\rangle d s$, for all $f \in E^{*}$, we write $v_{D}=\int_{D} u(s) d s$,

(b) Bochner integrable if there exists a sequence of countable-valued functions $\left\{u_{n}\right\}$ converging almost everywhere on $[a, b]$ such that $\lim _{n \rightarrow \infty} \int_{a}^{b}\left\|u_{n}(s)-u(s)\right\| d s=0$.

We note that every Bochner integrable function is Pettis integrable (see [14]).

Definition 1.3. The Hausdorff measure of weak noncompactness $\beta$ : $\mathscr{B} \rightarrow \mathrm{R}^{+}$and the Kuratowski measure of noncompactness $\alpha: \mathscr{B} \rightarrow \mathrm{R}^{+}$ are defined as follows: for each $U \in \mathscr{B}$,

(i) $\beta(U)=\inf \{\varepsilon>0: \exists K=$ weakly compact subset of $E, U \subseteq K+$ $\left.\varepsilon B_{1}\right\}$,

(ii) $\alpha(U)=\inf \{\varepsilon>0: U$ admits a finite cover of sets with diameter $<\varepsilon\}$.

For more details of $\beta$ and $\alpha$ we refer the reader to [1], [8]. 
Definition 1.4. By a Kamke function we mean a function $w: I \times \mathrm{R}^{+} \rightarrow$ $\mathrm{R}^{+}$such that:

(i) $w$ is a Carathéodory function,

(ii) for all $t \in I ; w(t, 0)=0$,

(iii) for any $c \in(0, b], u \equiv 0$ is the only absolutely continuous function on $[0, c]$ which satisfies $\dot{u}(t) \leq w(t, u(t))$ a.e. on $[0, c]$ and such that $u(0)=0$.

Definition 1.5. A continuous function $x:[-d, T] \rightarrow E_{w}$ is called a weak solution of problem $(\mathrm{P})$ if, for some $\xi \in C([-d, 0], E)$,

$$
x=\xi \quad \text { on } \quad[-d, 0]
$$

and

$$
x(t)=G(t, 0) \xi(0)+\int_{0}^{t} G(t, s) f(s, x(s)) d s \quad \text { for all } \quad t \in I .
$$

LEMMA 1.6. Let $\mathscr{F}$ be a continuous mapping from a compact interval I to $\mathscr{L}(E)$ and $\mathscr{U}$ be a bounded subset of $E$, then

$$
\gamma\left(\bigcup_{t \in I} \mathscr{F}(t) \mathscr{U}\right) \leq \sup _{t \in I}\|\mathscr{F}(t)\| \gamma(\mathscr{U})
$$

Proof. $\mathscr{U}$ is bounded, so $\exists c>0 ;\|\mathscr{U}\|=\sup \{\|u\|: u \in \mathscr{U}\} \leq c$. From the continuity of $\mathscr{F}$, for $\varepsilon>0$ there exists $\delta>0$ such that if $\bar{P}=$ $\left\{x_{0}, x_{1}, x_{2}, \ldots, x_{n}\right\}$ is a partition of $I$, that is, $a=x_{0}<x_{1}<x_{2}<\cdots<$ $x_{n}=b$ with $\|P\|=\sup \left\{\left|x_{i+1}-x_{i}\right|: i=0,1,2, \ldots, n-1\right\}<\delta$, then $\left\|\mathscr{F}\left(x_{i+1}\right)-\mathscr{F}\left(x_{i}\right)\right\|<\frac{\varepsilon}{c}$. Since $B_{1}$ is the closed unit ball in $E$, there exists a weakly compact subset $\mathscr{K}$ of $E$ such that $\mathscr{U} \subset \mathscr{K}+\frac{(\gamma(\mathscr{U})+\varepsilon)}{\gamma\left(B_{1}\right)} B_{1}$. But for each $t \in I_{i}=\left[x_{i}, x_{i+1}\right], \mathscr{F}(t) \mathscr{U} \subset\left\{\mathscr{F}(t) u-\mathscr{F}\left(t_{i+1}\right) u: u \in \mathscr{U}\right\}+\mathscr{F}\left(t_{i+1}\right) \mathscr{U}$ and $\left\|\mathscr{F}(t)-\mathscr{F}\left(t_{i+1}\right)\right\|\|\mathscr{U}\|<\frac{\varepsilon}{c} \cdot c=\varepsilon$. Hence $\left\{\mathscr{F}(t) u-\mathscr{F}\left(t_{i+1}\right) u: u \in \mathscr{U}\right\} \subset$ $\varepsilon B_{1}$ and $\mathscr{F}(t) \mathscr{U} \subseteq \varepsilon B_{1}+\mathscr{F}\left(t_{i+1}\right) \mathscr{U}$. Therefore

$$
\begin{aligned}
\bigcup_{t \in I} \mathscr{F}(t) \mathscr{U} & =\bigcup_{t=0}^{n-1} \bigcup_{t \in I} \mathscr{F}(t) \mathscr{U} \\
& \subseteq \varepsilon B_{1}+\bigcup_{t=0}^{n-1} \bigcup_{t \in I} \mathscr{F}(t) \mathscr{U} \subseteq \varepsilon B_{1}+\bigcup_{t=0}^{n-1}\left(\varepsilon B_{1}+\mathscr{F}\left(t_{i+1}\right) \mathscr{U}\right)
\end{aligned}
$$




$$
\begin{aligned}
& \subseteq 2 \varepsilon B_{1}+\bigcup_{t=0}^{n-1} \mathscr{F}\left(t_{i+1}\right)\left(\mathscr{K}+\frac{(\gamma(\mathscr{U})+\varepsilon)}{\gamma\left(B_{1}\right)} B_{1}\right) \\
& \subseteq 2 \varepsilon B_{1}+\bigcup_{t=0}^{n-1} \mathscr{F}\left(t_{i+1}\right) \mathscr{K}+\bigcup_{t=0}^{n-1} \mathscr{F}\left(t_{i+1}\right) \frac{(\gamma(\mathcal{U})+\varepsilon)}{\gamma\left(B_{1}\right)} B_{1} \\
& \subseteq 2 \varepsilon B_{1}+\bigcup_{t=0}^{n-1} \mathscr{F}\left(t_{i+1}\right) \mathscr{H}+\sup _{t \in I}\|\mathscr{F}(t)\| \frac{(\gamma(\mathcal{U})+\varepsilon)}{\gamma\left(B_{1}\right)} B_{1} .
\end{aligned}
$$

Moreover

$$
\begin{aligned}
\gamma\left(\bigcup_{t \in I} \mathscr{F}(t) \mathcal{U}\right) & \leq \gamma\left(2 \varepsilon B_{1}+\bigcup_{t=0}^{n-1} \mathscr{F}\left(t_{i+1}\right) \mathscr{K}+\sup _{t \in I}\|\mathscr{F}(t)\| \frac{(\gamma(\mathscr{U})+\varepsilon)}{\gamma\left(B_{1}\right)} B_{1}\right) \\
& \leq 2 \varepsilon \gamma\left(B_{1}\right)+\gamma\left(\sup _{t \in I}\|\mathscr{F}(t)\| \frac{(\gamma(\mathscr{U})+\varepsilon)}{\gamma\left(B_{1}\right)} B_{1}\right) \\
& \leq 2 \varepsilon \gamma\left(B_{1}\right)+\sup _{t \in I}\|\mathscr{F}(t)\| \frac{(\gamma(\mathcal{U})+\varepsilon)}{\gamma\left(B_{1}\right)} \gamma\left(B_{1}\right) \\
& \leq 2 \varepsilon \gamma\left(B_{1}\right)+\sup _{t \in I}\|\mathscr{F}(t)\|(\gamma(\mathscr{U})+\varepsilon)
\end{aligned}
$$

where $\bigcup_{t=0}^{n-1} \mathscr{F}\left(t_{i+1}\right) \mathscr{K}$ is weakly compact. Since $\varepsilon$ is arbitrary the result follows.

Lemma 1.7 ([3]). Let $Y$ and $E$ be two Banach spaces, $P_{f c}(Y)$ be the set of all closed and convex subsets of $Y$ and $F: E \rightarrow P_{f c}(Y)$ be weakly sequentially upper hemicontinuous. Further let $\left(x_{n}\right)_{n \in \mathrm{N}} \subset C(I, E), x_{n}(t) \rightarrow x_{0}(t)$ weakly a.e. on $I$ and $\left(y_{n}\right)_{n \in \mathrm{N} \cup\{0\}} \subset L^{1}(I, E), y_{n} \rightarrow y_{0}$ weakly. Suppose that there exists $a \in L^{1}(I, \mathrm{R})$ such that $\|F(x)\| \leq a(t)$ for all $x \in C(I, E)$ and $y_{n}(t) \in$ $F\left(x_{n}(t)\right)$ a.e. on I. Then $y_{0}(t) \in F\left(x_{0}(t)\right)$ a.e. on $I$.

LEMmA 1.8 ([18], [1]). If $\gamma: \mathscr{B} \rightarrow \mathrm{R}^{+}$satisfies conditions $\left(\mathrm{M}_{2}\right),\left(\mathrm{M}_{4}\right)$ and $\left(\mathrm{M}_{6}\right)$ then, for any nonempty $U \in \mathscr{B}$,

$$
\gamma(U) \leq \gamma\left(B_{1}\right) \alpha(U) \leq 2 \gamma\left(B_{1}\right) \beta(U)
$$

LEMMA 1.9 ([21], [17]). If $\gamma$ is a measure of weak (strong) noncompactness and $A \subset C_{w}(I, E)$ is a family of strongly equicontinuous functions, then

$$
\gamma(A(I))=\sup \{\gamma(A(t)): t \in I\} .
$$


If for each $t \in \mathrm{R}^{+}, A(t) \in \mathscr{L}(E)$ and $\dot{x}(t)$ denotes the weak derivative of $x$ at $t$, then we consider the differential equation

$$
\dot{x}(t)=A(t) x(t) .
$$

Let $E$ be the direct sum of $\mathscr{E}_{0}$ and $\mathscr{E}_{1}$, where

$$
\mathscr{E}_{0}=\left\{x_{0} \in E: \exists \text { a bounded weak solution } x \text { of }(1) \text { and } x(0)=x_{0}\right\}
$$

is closed and has a closed complement $\mathscr{E}_{1}$.

Let $G \in C\left(\mathrm{R}^{+} \times \mathrm{R}^{+}, E\right)$ be the Green function corresponding to (1):

$$
G(t, s)= \begin{cases}S(t) P S^{-1}(s) & \text { if } 0 \leq s \leq t \\ -S(t)(i d-P) S^{-1}(s) & \text { if } 0 \leq t \leq s,\end{cases}
$$

where $S: \mathrm{R}^{+} \rightarrow \mathscr{L}(E)$ is a solution of the differential equation

$$
\dot{S}(t)=A(t) S(t), \quad S(0)=i d,
$$

and $P$ is the projection of $E$ onto $\mathscr{E}_{0}$; hence $P\left(\mathscr{E}_{1}\right)=\{0\}$.

\section{Existence results for problem (P)}

In this section we shall consider the nonlinear differential equation

$$
\dot{x}(t)=A(t) x(t)+f(t, x(t)), \quad t \in \mathrm{R}^{+} .
$$

This problem was studied by many authors (see, for instance, [5], [19], [6], [16], [10]). The next theorem is a generalization of Theorem 8 in [13]. Moreover we use here a general weak noncompactness measure, in contrast with the Hausdorff noncompactness measure used in [10]; hence, the result below is at the same time a generalization of Theorem 5 in [10].

Theorem 2.1. Let $A: \mathrm{R}^{+} \rightarrow \mathscr{L}(E)$ be strongly measurable and Bochner integrable on every subinterval I of $\mathrm{R}^{+}$. Let $\gamma$ be a weak measure of noncompactness, for each $t \in \mathrm{R}^{+}$, let $G(t,.) \in \mathscr{M}^{\prime}$ with $\|G(t, .)\|_{\mathscr{M}} \leq c$ where $c>0$. Let $f$ be a continuous function from $\mathbf{R}^{+} \times E_{w}$ to $E_{w}$ and $m: \mathbf{R}^{+} \rightarrow \mathbf{R}^{+}$belongs to $\mathcal{M}^{\prime}$ such that $\|f(t, x)\| \leq m(t)$ for every $(t, x) \in \mathrm{R}^{+} \times B_{r}$. Assume that $c\|m\|_{\mathscr{M}}<r$ and for each $T, \varepsilon>0$ there exists a closed subset $I_{\varepsilon}$ of $I$ with $\lambda\left(I-I_{\varepsilon}\right)<\varepsilon$ such that for any nonempty bounded subset $U$ of $E$ one has $\beta(f(J \times U)) \leq \sup _{t \in J} w(t, \beta(U))$, for any compact subset $J$ of $I_{\varepsilon}$. Then, for each $x_{0} \in \mathscr{E}_{0}$ such that $\left\|x_{0}\right\| \leq \frac{r-c\|m\|_{.}}{\|G(t, 0)\|}$, there exists a bounded weak solution of $(\mathrm{P})$. 
Proof. Let

$$
\begin{aligned}
S=\left\{x \in C_{w}\left(\mathrm{R}^{+}, E\right): \| x\right. & (t)-x(\tau) \| \\
& \left.\leq r \int_{t}^{\tau}|A(s)| d s+\int_{t}^{\tau} m(s) d s, 0 \leq t \leq \tau\right\} .
\end{aligned}
$$

From (2) and by results from [20] there exists a positive number $d$ such that $\|G(t, 0)\| \leq d$. Let $x_{0} \in \mathscr{E}_{0}$ with $\left\|x_{0}\right\| \leq \frac{r-c\|m\|_{\mathscr{M}}}{d}$. Then $G(t, 0) x_{0}$ is a solution of (1) and $\left\|G(t, 0) x_{0}\right\| \leq d\left\|x_{0}\right\| \leq r-c\|m\|_{\mu}$. If $\phi$ is defined by

$\phi(x)(t)=G(t, 0) x_{0}+\int_{0}^{\infty} G(t, s) f(s, x(s)) d s \quad$ for $\quad t \in \mathrm{R}^{+}$and $x \in S$,

then

$$
\|\phi(x)(t)\| \leq d\left\|x_{0}\right\|+c\|m\|_{M} \leq r .
$$

Since $y=\phi(x)$ is a weak solution of the equation $\dot{y}(t)=A(t) y(t)+f(t, x(t))$, we have

$$
\begin{aligned}
\|\phi(x)(t)-\phi(x)(\tau)\| & \leq \int_{t}^{\tau}\|A(s) \phi(x)(s)+f(s, x(s))\| d s \\
& \leq r \int_{t}^{\tau}|A(s)| d s+\int_{t}^{\tau} m(s) d s .
\end{aligned}
$$

Therefore $\phi$ is a continuous mapping from $S$ into $S$ [4]. Let $\left(x_{n}\right)_{n \in \mathrm{N} \cup\{0\}}$ be a sequence such that $\phi\left(x_{n}\right)=x_{n+1}$ with $x_{0}$ is an arbitrary element in $S$. Thus $D \subset S$ and, from $\left(\mathrm{M}_{4}\right), \gamma(D)=\gamma(\phi(D))$. If $G$ is the set of all limit points of the sequence $\left(x_{n}\right)$, then $\phi(G)=G$. Put $R(X)=\operatorname{conv} \phi(X)$ for $X \subset S$ and consider the family $\Omega$ of all subsets $X$ of $S$ such that $G \subset X$ and $R(X) \subset X$. Now $S \in \Omega$ and so $\Omega \neq \emptyset$. Let $V$ be the intersection of all sets of the family $\Omega$. Then $V \in \Omega$. Moreover the mapping $t \rightarrow \gamma(\phi(V)(t))$ is absolutely continuous. Assume that $t \geq 0$ and $\varepsilon>0$ thus from the assumptions on the function $m$ we can find $T_{0} \geq t$ such that $\left\|m \chi_{\left[T_{0}, \infty[\right.}\right\|_{\mu}<\frac{\varepsilon}{2 c}$. If we put $I_{0}:=\left[0, T_{0}\right]$, then by the Scorza-Dragoni theorem there exists a closed subset $I_{\varepsilon}$ of $I_{0}$ such that $\lambda\left(I_{0}-I_{\varepsilon}\right)<\delta$ and the function $w$ is uniformly continuous on $I_{\varepsilon} \times\left[0,2 T_{0}\right]$. From our last assumption, we can find a closed subset $J_{\varepsilon}$ of $I_{0}$ such that $\lambda\left(I_{0}-J_{\varepsilon}\right)<\delta$ and such that for any compact subset $\mathscr{C}$ of $J_{\varepsilon}$ and any bounded subset $Z$ of $E$,

$$
\gamma(f(\mathscr{C} \times Z)) \leq \sup _{s \in \mathscr{C}} w(s, \gamma(Z)) .
$$

Since $\phi$ is continuous and $w$ is Carathéodory we can find a closed subset $I_{\varepsilon}$ of $I, \delta>0, \eta>0(\eta<\delta)$ such that if $s_{1}, s_{2} \in I_{\varepsilon}$ and $r_{1}, r_{2} \in\left[0,2 T_{0}\right]$ 
satisfy $\left|s_{1}-s_{2}\right|<\delta,\left|r_{1}-r_{2}\right|<\delta$, then $\left|w\left(s_{1}, r_{1}\right)-w\left(s_{2}, r_{2}\right)\right|<\varepsilon$ and if $\left|s_{1}-s_{2}\right|<\eta$, then $\left|\gamma\left(V\left(s_{1}\right)\right)-\gamma\left(V\left(s_{2}\right)\right)\right|<\delta$. Let us fix $\tau$ such that $0 \leq t \leq \tau \leq T$ and consider the partition, to $[t, \tau], t=t_{0}<t_{1}<\cdots<$ $t_{m}=\tau$ such that $t_{i}-t_{i-1}<\eta$ for $i=1, \ldots, n$. Let $T_{i}=J_{\varepsilon} \cap\left[t_{i-1}, t_{i}\right] \cap I_{\varepsilon}$, $P=\sum_{i=1}^{m} T_{i}=[t, \tau] \cap J_{\varepsilon} \cap I_{\varepsilon}$ and $Q=[t, \tau]-P$. Since $G(t,$.$) is uniformly$ continuous on $P$, we can find $\eta^{\prime}>0\left(\eta^{\prime}<\delta\right)$ such that if $r_{1}, r_{2} \in P$ and $\left|r_{1}-r_{2}\right|<\eta^{\prime}$, then

$$
\left\|G\left(t, r_{1}\right)-G\left(t, r_{2}\right)\right\|<\varepsilon
$$

and we can find $s_{i}$ in $T_{i}$ with

$$
\sup _{s \in T_{i}}\|G(t, s)\|=\left\|G\left(t, s_{i}\right)\right\| .
$$

Let $S_{i}=\left\{x(t): x \in S, t \in T_{i}\right\}$. In virtue of Lemma 1.6, Lemma 1.9, the mean value theorem and Lemma 1.8 if $\rho(t):=\gamma(V(t))$ we get

$$
\begin{aligned}
\rho(\tau)-\rho(t) & \leq \gamma \int_{t}^{\tau} G(t, s) f(s, V(s)) d s \\
& \leq 2 \gamma\left(B_{1}\right) \int_{t}^{\tau}\|G(t, s)\| w(s, \rho(s)) d s .
\end{aligned}
$$

Therefore $\dot{\rho}(t) \leq c w(t, \rho(t))$ a.e. [12] and since $\rho(0)=0$, then $\rho \equiv 0$ and so $\bar{V}^{w}$ is weakly compact in $C_{w}\left(\mathrm{R}^{+}, E\right)$. But $V$ is closed, hence it is a convex and compact subset in $C_{w}\left(\mathrm{R}^{+}, E\right)$. From the Schauder-Tichonov theorem, since $\phi$ is a continuous mapping from $V$ to $V$, there is a fixed point $y$ of $\phi$ such that $y$ is the desired weak solution of $(\mathrm{P})$ and satisfies $\sup _{t \in \mathrm{R}^{+}}\|y(t)\| \leq r$.

In the following theorem we will deal with the differential equation

$$
\dot{x}(t)=L(t) x(t)+f^{\prime}(t, x(t)), \quad t \in I
$$

where $f^{\prime}: I \times B_{r} \rightarrow E$ is a Carathéodory function, $L: I \rightarrow \mathscr{L}(E)$ is strongly measurable and Bochner integrable operator on $I$ and $\gamma$ is a measure of strong noncompactness. We get a generalization of Theorem 2 in [26] and Theorem 9 in [13].

THEOREM 2.2. In the setting of Theorem 2.1 we replace the function $f$ by $f^{\prime}$ such that for each $x \in B_{r}, f^{\prime}(I \times\{x\})$ is separable; the function $m$ by $m^{\prime} \in L^{1}\left(I, \mathrm{R}^{+}\right)$and the operator $A$ by $L$. Then problem $\left(\mathrm{P}^{\prime}\right)$ has a solution.

Proof. Let

$$
\begin{aligned}
S=\{x \in C(I, E): \| x(t) & -x(\tau) \| \\
\leq & \left.r \int_{t}^{\tau}|A(s)| d s+\int_{t}^{\tau} m^{\prime}(s) d s, 0 \leq t \leq \tau\right\} .
\end{aligned}
$$


Suppose that the mapping $\phi: S \rightarrow S$ is defined by

$$
\phi(x)(t)=G(t, 0) x_{0}+\int_{0}^{t} G(t, s) f(s, x(s)) d s \quad \text { for } t \in I \text { and } x \in S .
$$

As in Theorem 2.1 we let $\left(x_{n}\right)_{n \in \mathrm{N} \cup\{0\}}$ be a sequence such that $\phi\left(x_{n}\right)=x_{n+1}$ where $x_{0}$ is an arbitrary element in $S, V=\left\{x_{n}: n=0,1,2, \ldots\right\}, V \subset S$, $\gamma(V)=\gamma(\phi(V))$ and $\rho(t)=\gamma(V(t))$. Then by the same argument we get

$$
\begin{aligned}
\rho(\tau)-\rho(t) & \leq \gamma\left(\int_{t}^{\tau} G(t, s) f(s, V(s)) d s\right) \\
& \leq \gamma\left(B_{1}\right) \int_{t}^{\tau}\|G(t, s)\| w(s, \rho(s)) d s,
\end{aligned}
$$

$\rho$ is differentiable a.e. on $I$ and $\rho \equiv 0$. Thus the closure of $V$ is compact in $C(I, E)$ and so we can find a subsequence $\left(x_{n_{k}}\right)$ of $\left(x_{n}\right)$ which converges to a limit $x$ in $C(I, E)$. Since $\left\|x_{n}-\phi\left(x_{n}\right)\right\| \rightarrow 0$ as $n \rightarrow \infty$ and $\phi$ is continuous, then $x=\phi(x)$ so as $x$ is the desired solution of $\left(\mathrm{P}^{\prime}\right)$ and $\|x\| \leq r$.

In the following theorem we let $h: I \times \mathrm{R}^{+} \rightarrow \mathrm{R}^{+}$be a Carathéodory function, such that for each bounded subset $Z$ of $I \times \mathrm{R}^{+}$there exists a function $\varphi: I \rightarrow \mathrm{R}^{+}$such that $h(t, s) \leq \varphi(t),(t, s) \in Z$ and $\varphi$ is integrable function on $[c, T]$ for each $c, 0<c \leq T$. Moreover we assume that the identically zero function is the only absolutely continuous function on $[0, c]$ which satisfies $\dot{u}(t)=h(t, u(t))$ a.e. on $[0, c]$ such that the right derivative $D_{+} u(0)$ of $u(t)$ at $t=0$ exists and $D_{+} u(0)=u(0)=0$.

We note that the assumptions on $h$ are weaker than that on a Kamke function $w$.

THEOREM 2.3. If we replace in the setting of Theorem 2.2 a Kamke function $w$ by a function $h$ and we suppose that $f^{\prime}$ is bounded and continuous, then problem $\left(\mathrm{P}^{\prime}\right)$ has a solution.

Proof. By the same argument as in Theorem 2.2 we get

$$
\begin{aligned}
\rho(\tau)-\rho(t) & \leq \gamma \int_{t}^{\tau} G(t, s) f(s, V(s)) d s \\
& \leq \gamma\left(B_{1}\right) \int_{t}^{\tau}\|G(t, s)\| h(s, \rho(s)) d s
\end{aligned}
$$

where $\rho(t)=\gamma(V(t))$. Since $f^{\prime}$ is a bounded function, we can find a constant $M>0$ such that $\left\|f^{\prime}(t, x)\right\| \leq M$ for each $(t, x) \in I \times B_{r}$. Let $\mathcal{N}: I \rightarrow \mathrm{R}$ be defined by $\mathcal{N}(t)=\sup _{\|x\|,\|y\| \leq M t}\left\|f^{\prime}(t, x)-f^{\prime}(t, y)\right\|$. We see that $\mathscr{N}$ is 
lower semicontinuous on ]0,T] and continuous at 0 [22]. Let $\varepsilon>0$ and $t_{0}$ be fixed in $I$. Then, there exist $x_{1}, y_{1} \in B_{r} ;\left\|x_{1}\right\|,\left\|y_{1}\right\| \leq M t$ such that

$$
\mathcal{N}\left(t_{0}\right)-\frac{\varepsilon}{2} \leq\left\|f^{\prime}\left(t_{0}, x_{1}\right)-f^{\prime}\left(t_{0}, y_{1}\right)\right\| .
$$

Moreover, $f^{\prime}$ is continuous. Thus $\exists \delta>0$ such that if $\left|t-t_{0}\right|<\delta,\left\|x_{1}-x\right\|<\delta$, $\left\|y_{1}-y\right\|<\delta$, we have

$$
\left\|f^{\prime}\left(t_{0}, x_{1}\right)-f^{\prime}(t, x)\right\|<\frac{\varepsilon}{4} \quad \text { and } \quad\left\|f^{\prime}\left(t_{0}, y_{1}\right)-f^{\prime}(t, y)\right\|<\frac{\varepsilon}{4} .
$$

From relations (4) and (5), we get

$$
\begin{aligned}
\mathcal{N}\left(t_{0}\right)-\frac{\varepsilon}{2} \leq & \left\|f^{\prime}\left(t_{0}, x_{1}\right)-f^{\prime}\left(t_{0}, y_{1}\right)\right\| \\
\leq & \left\|f^{\prime}\left(t_{0}, x_{1}\right)-f^{\prime}(t, x)\right\| \\
& \quad+\left\|f^{\prime}(t, x)-f^{\prime}(t, y)\right\|+\left\|f^{\prime}(t, y)-f^{\prime}\left(t_{0}, y_{1}\right)\right\| \\
& \leq\left\|f^{\prime}(t, x)-f^{\prime}(t, y)\right\|+\frac{\varepsilon}{2},
\end{aligned}
$$

and so,

$$
\mathcal{N}\left(t_{0}\right)-\varepsilon \leq\left\|f^{\prime}(t, x)-f^{\prime}(t, y)\right\| .
$$

Thus, for each $t$ with $\left|t-t_{0}\right|<\delta$, there exist $x_{1}, y_{1}$ with $\left\|x_{1}\right\|,\left\|y_{1}\right\| \leq M t$ such that $\mathcal{N}\left(t_{0}\right)-\varepsilon \leq\left\|f^{\prime}\left(t, x_{1}\right)-f^{\prime}\left(t, y_{1}\right)\right\| \leq \mathcal{N}(t)$. We conclude that $\mathcal{N}$ is lower semicontinuous. Moreover from the continuity of $f^{\prime}, \mathcal{N}$ is continuous at 0 . Consequently we can say that $\left\|\int_{t}^{\tau} f^{\prime}(s, x(s))-\int_{t}^{\tau} f^{\prime}(s, y(s)) d s\right\| \leq$ $\int_{t}^{\tau} \mathcal{N}(s) d s$ for each $x, y \in V$. Then from relation (3) we have

$$
\begin{array}{r}
\rho(\tau)-\rho(t) \\
\leq \min \left(\int_{t}^{\tau}\|G(t, s)\| \mathcal{N}(s) d s, \gamma\left(B_{1}\right) \int_{t}^{\tau}\|G(t, s)\| h(s, \rho(s)) d s\right),
\end{array}
$$

where $0<t \leq \tau \leq T$. Therefore $\rho$ is an absolutely continuous function on $I$ and so

$$
\dot{\rho}(t) \leq \min (\|G(t, s)\| \mathcal{N}(t),\|G(t, s)\| h(t, \rho(t))), \quad \text { a.e. on } \quad I .
$$

Thus $\rho \equiv 0$ on $I$, see Lemma 1 in [22]. We can complete the proof as in the proof of Theorem 2.2. 


\section{Existence results for problem (Q)}

We consider the problem

$$
\dot{x}(t)=L(t) x(t)+f^{d}\left(t, \theta_{t} x\right), \quad t \in I .
$$

Let $B_{r}=\{x \in E:\|x\| \leq r\}, L(t) \in \mathscr{L}(E)$ and for $t \in I$ we define $\theta_{t} x(s)=x(t+s)$ for all $s \in[-d, 0]$. We assume that $C\left([-d, 0], B_{r}\right)$ is the Banach space of continuous functions from $[-d, 0]$ into $B_{r}$ and $f^{d}$ : $I \times C\left([-d, 0], B_{r}\right) \rightarrow E$.

In the following theorem we deal with problem $(\mathrm{Q})$ and we have a generalization of Theorem 2.1.

THEOREM 3.1. If we replace in the setting of Theorem 2.1 the function $f$ by $f^{d}$; the function $m$ by $m^{\prime} \in L^{1}\left(I, \mathrm{R}^{+}\right)$and the operator $A$ by $L$, then problem (Q) has a weak solution.

Proof. We apply some methods for functional equations similar to those of [10]. For any arbitrary $n \in \mathrm{N}$, we define $\gamma_{1}:\left[-d, \frac{T}{n}\right] \times E \rightarrow E$ by

$$
\gamma_{1}(t, x)= \begin{cases}\xi(t) & \text { if } t \in[-d, 0] \\ \xi(0)+n t(x-\xi(0)) & \text { if } t \in\left[0, \frac{T}{n}\right]\end{cases}
$$

and also we define $f_{1}:\left[0, \frac{T}{n}\right] \times E \rightarrow E$ by $f_{1}(t, x)=f^{d}\left(t, \theta_{\frac{T}{n}}\left(\gamma_{1}(., x)\right)\right)$. Arguing as in the proof of Theorem 2.1, there is a continuous function $y_{1}$ such that $y_{1}=\xi$ on $[-d, 0]$ and for each $t \in\left[0, \frac{T}{n}\right]$

$$
y_{1}(t)=G(t, 0) \xi(0)+\int_{0}^{t} G(t, s) f_{1}\left(s, y_{1}(s)\right) d s .
$$

Moreover $\sup _{t \in\left[0, \frac{T}{n}\right]}\left\|y_{1}(t)\right\| \leq r$. Set $k^{\prime}=k-1$. By induction, for each $k \in\{2,3, \ldots, n\}$, there exists a bounded function $y_{k^{\prime}}$ such that $y_{k^{\prime}}=\xi$ on $[-d, 0]$ and for each $t \in\left[0, \frac{k^{\prime} T}{n}\right]$

$$
y_{k^{\prime}}(t)=G(t, 0) \xi(0)+\int_{0}^{t} G(t, s) f_{k^{\prime}}\left(s, y_{k^{\prime}}(s)\right) d s,
$$

where $f_{k^{\prime}}(t, x)=f^{d}\left(t, \theta_{\frac{k^{\prime} T}{n}} \gamma_{k^{\prime}}(., x)\right)$. Assume that $\gamma_{k}:\left[-d, \frac{k T}{n}\right] \times E \rightarrow E$ is such that

$$
\gamma_{k}(t, x)= \begin{cases}y_{k^{\prime}}(t) & \text { if } t \in\left[-d, \frac{k^{\prime} T}{n}\right] \\ y_{k^{\prime}}\left(\frac{k^{\prime} T}{n}\right)+n\left(t-\frac{k^{\prime} T}{n}\right)\left(x-y_{k^{\prime}}\left(\frac{k^{\prime} T}{n}\right)\right) & \text { if } t \in\left[\frac{k^{\prime} T}{n}, \frac{k T}{n}\right] .\end{cases}
$$


Thus if $f_{k}:\left[\frac{k^{\prime} T}{n}, \frac{k T}{n}\right] \times E \rightarrow E$ is defined by $f_{k}(t, x)=f^{d}\left(t, \theta_{\frac{k T}{n}}\left(\gamma_{k}(., x)\right)\right)$, then we have a continuous function $y_{k}$ defined on $\left[\frac{k^{\prime} T}{n}, \frac{k T}{n}\right]$ by

$$
y_{k}(t)=G\left(t, \frac{k^{\prime} T}{n}\right) y_{k^{\prime}}\left(\frac{k^{\prime} T}{n}\right)+\int_{\frac{k^{\prime} T}{n}}^{t} G(t, s) f_{k}\left(s, y_{k}(s)\right) d s
$$

Further, for $0 \leq s \leq r \leq t, G(t, s) G(s, r)=G(t, r)$ and for each $t \in$ $\left[\frac{k^{\prime} T}{n}, \frac{k T}{n}\right]$ we have

$$
y_{k^{\prime}}\left(\frac{k^{\prime} T}{n}\right)=G\left(\frac{k^{\prime} T}{n}, 0\right) \xi(0)+\int_{0}^{\frac{k^{\prime} T}{n}} G\left(\frac{k^{\prime} T}{n}, s\right) f_{k^{\prime}}\left(s, y_{k^{\prime}}(s)\right) d s .
$$

Hence

$$
\begin{aligned}
y_{k}(t)=G\left(t, \frac{k^{\prime} T}{n}\right) G\left(\frac{k^{\prime} T}{n}, 0\right) \xi(0)+\int_{0}^{\frac{k^{\prime} T}{n}} G\left(t, \frac{k^{\prime} T}{n}\right) G\left(\frac{k^{\prime} T}{n}, s\right) f_{k^{\prime}}\left(s, y_{k^{\prime}}(s)\right) d s \\
\quad+\int_{\frac{k^{\prime} T}{n}}^{t} G(t, s) f_{k}(s, x(s)) d s \\
=G(t, 0) \xi(0)+\int_{0}^{\frac{k^{\prime} T}{n}} G(t, s) f_{k^{\prime}}\left(s, y_{k^{\prime}}(s)\right) d s \\
\quad+\int_{\frac{k^{\prime} T}{n}}^{t} G(t, s) f_{k}\left(s, y_{k}(s)\right) d s \\
=G(t, 0) \xi(0)+\int_{0}^{t} G(t, s) g_{k}\left(s, y_{k}(s)\right) d s,
\end{aligned}
$$

where

$$
g_{k}\left(t, y_{k}(t)\right)= \begin{cases}f_{k^{\prime}}\left(t, y_{k^{\prime}}(t)\right) & \text { if } t \in\left[0, \frac{k^{\prime} T}{n}\right] \\ f_{k}\left(t, y_{k}(t)\right) & \text { if } t \in\left[\frac{k^{\prime} T}{n}, \frac{k T}{n}\right]\end{cases}
$$

Consequently, for all $n \in \mathrm{N}$, we have a continuous bounded function $v_{n}$ such that $v_{n}=\xi$ on $[-d, 0]$ and for each $t \in I, \frac{k^{\prime} T}{n} \leq t \leq \frac{k T}{n}$ for some $k \in$ $\{1,2,3, \ldots, n\}$, we have

$$
v_{n}(t)=G(t, 0) \xi(0)+\int_{0}^{t} G(t, s) h_{n}(s) d s
$$


where $h_{n}(t)=f^{d}\left(t, \theta_{\frac{k T}{n}} \gamma_{k}\left(., v_{n}(t)\right)\right)$. Let $t_{1}, t_{2} \in I$ and $t_{1}<t_{2}$. Then

$$
\begin{aligned}
& \left\|v_{n}\left(t_{1}\right)-v_{n}\left(t_{2}\right)\right\| \\
& \leq\left\|G\left(t_{1}, 0\right)-G\left(t_{2}, 0\right)\right\|\|\xi(0)\|+\int_{0}^{t_{1}}\left\|G\left(t_{1}, s\right)-G\left(t_{2}, s\right)\right\|\left\|h_{n}\left(s, v_{n}(s)\right)\right\| d s \\
& \quad+\int_{t_{1}}^{t_{2}}\left\|G\left(t_{2}, s\right)\right\|\left\|h_{n}\left(s, v_{n}(s)\right)\right\| d s \\
& \leq\left\|G\left(t_{1}, 0\right)-G\left(t_{2}, 0\right)\right\|\|\xi(0)\|+\int_{0}^{t_{1}}\left\|G\left(t_{1}, s\right)-G\left(t_{2}, s\right)\right\|\left\|m^{\prime}(s)\right\| d s \\
& \quad+c \int_{t_{1}}^{t_{2}}\left\|m^{\prime}(s)\right\| d s,
\end{aligned}
$$

since $v_{n}=\xi$ on $[-d, 0]$ and for all $s \in I G(., s)$ is uniformly continuous, then $A$ is equicontinuous in $C([-d, T], E) . \gamma(A(t))=\gamma\left(\left\{v_{n}(t): n \in \mathrm{N}\right\}\right)$ is such that $\gamma(A(0))=0$ and, as in the proof of Theorem 2.1, $\gamma(A(t))=0$ for all $t \in I$. Thus by Ascoli's theorem, the sequence $\left\{v_{n}: n \in \mathrm{N}\right\}$ converges uniformly to a function $v$ which belongs to $C([-d, T], E)$ such that $y=\xi$ on $[-d, 0]$. But $\gamma\left(\left\{h_{n}(t): n \in \mathrm{N}\right\}\right)=0$ and so $\left\{h_{n}(t): n \in \mathrm{N}\right\}$ is relatively compact. Let $\mathscr{F}(t)=\overline{\operatorname{conv}}\left\{h_{n}(t): n \in \mathrm{N}\right\}$. Thus $\mathscr{F}(t)$ is nonempty convex and compact. Moreover $\delta_{\mathscr{F}}^{1}=\left\{l \in L^{1}(I, E): l(t) \in \mathscr{F}(t)\right\}$ is nonempty convex and weakly compact. Therefore, there exists a subsequence $\left(h_{n_{k}}\right)$ of $\left(h_{n}\right)$ such that $h_{n_{k}} \rightarrow l$ weakly, $l \in \delta_{\mathscr{F}}^{1}$. Thus $\left\{v_{n}: n \in \mathrm{N}\right\}$ tends weakly to $v(t):=G(t, 0) \xi(0)+\int_{0}^{t} G(t, s) l(s) d s$. Now $v$ is uniformly continuous on $[-d, 0]$ and for each $t \in I$, there exists $n>\frac{T}{d}$ with $t \in\left[\frac{k^{\prime} T}{n}, \frac{k T}{n}\right]$ for $k \in\{1,2, \ldots, n-1\}$. Hence

$$
\begin{aligned}
& \left\|\theta_{\frac{k T}{n}} \gamma_{k}\left(., v_{n}(t)\right)-\theta_{t} v\right\| \\
& \leq \sup _{s \in\left[-d,-\frac{T}{n}\right]}\left[\left\|\gamma_{k}\left(\frac{k T}{n}+s, v_{n}(t)\right)-v\left(\frac{k T}{n}+s\right)\right\|+\left\|v\left(\frac{k T}{n}+s\right)-v(t+s)\right\|\right] \\
& +\sup _{s \in\left[-\frac{T}{n}, 0\right]}\left[\left\|v_{n}\left(\frac{k^{\prime} T}{n}\right)+n\left(\frac{k T}{n}+s-\frac{k^{\prime} T}{n}\right)\left(v_{n}(t)-v_{n}\left(\frac{k^{\prime} T}{n}\right)\right)-v\left(\frac{k T}{n}+s\right)\right\|\right. \\
& \left.+\left\|v\left(\frac{k T}{n}+s\right)-v(t+s)\right\|\right] \\
& \leq \sup _{s \in\left[-d,-\frac{T}{n}\right]}\left[\left\|v_{n}\left(\frac{k T}{n}+s\right)-v\left(\frac{k T}{n}+s\right)\right\|+\left\|v\left(\frac{k T}{n}+s\right)-v(t+s)\right\|\right] \\
& +\sup _{s \in\left[-\frac{T}{n}, 0\right]}\left[T\left\|\left(v_{n}(t)-v_{n}\left(\frac{k^{\prime} T}{n}\right)\right)\right\|+\left\|v_{n}\left(\frac{k^{\prime} T}{n}\right)-v\left(\frac{k T}{n}+s\right)\right\|\right. \\
& \left.+\left\|v\left(\frac{k T}{n}+s\right)-v(t+s)\right\|\right]
\end{aligned}
$$


as $n \rightarrow \infty$. So from Lemma 1.7, problem (Q) has a weak solution $v$.

In the following theorem we use a measure of strong noncompactness $\gamma$ so we have a generalization of Theorem 3.1 and an improvement to Theorem 2 in [26] and Theorem 9 in [13].

THEOREM 3.2. In the setting of Theorem 2.2 if we replace the function $f^{\prime}$ by $f^{d}$ such that for all $\varphi \in C\left([-d, 0], B_{r}\right) f^{d}(I \times\{\varphi\})$ is separable, then problem $(\mathrm{Q})$ has a solution.

Proof. For $n \in \mathrm{N}$ we define $\gamma_{1}:\left[-d, \frac{T}{n}\right] \times E \rightarrow E$, as in the proof of Theorem 3.1, by

$$
\gamma_{1}(t, x)= \begin{cases}\xi(t) & \text { if } t \in[-d, 0] \\ \xi(0)+n t(x-\xi(0)) & \text { if } t \in\left[0, \frac{T}{n}\right]\end{cases}
$$

and $f_{1}:\left[0, \frac{T}{n}\right] \times E \rightarrow E$ by $f_{1}(t, x)=f^{d}\left(t, \theta_{\frac{T}{n}}\left(\gamma_{1}(., x)\right)\right)$. By Theorem 2.2 there exists a continuous function $y_{1}$ such that $y_{1}{ }^{n}=\xi$ on $[-d, 0]$ and for each $t \in\left[0, \frac{T}{n}\right]$

$$
y_{1}(t)=G(t, 0) \xi(0)+\int_{0}^{t} G(t, s) f_{1}\left(s, y_{1}(s)\right) d s .
$$

Then we can construct, for each $n \in \mathrm{N}$, a continuous bounded function $v_{n}$ such that $v_{n}=\xi$ on $[-d, 0]$ and for each $t \in I v_{n}$ is defined by

$$
v_{n}(t)=G(t, 0) \xi(0)+\int_{0}^{t} G(t, s) h_{n}(s) d s,
$$

where $h_{n}(t)=f^{d}\left(t, \theta_{\frac{k T}{n}} \gamma_{k}\left(., v_{n}(t)\right)\right)$ with $k \in\{1,2,3, \ldots, n\}$ and $\frac{(k-1) T}{n} \leq$ $t \leq \frac{k T}{n}$. We can complete the proof as in the proof of Theorem 3.1.

In the next theorem we let $h: I \times \mathrm{R}^{+} \rightarrow \mathrm{R}^{+}$be a Carathéodory function. Also for each bounded subset $Z$ of $I \times \mathrm{R}^{+}$we suppose that there exists a function $m: I \rightarrow \mathrm{R}^{+}$such that $h(t, s) \leq m(t),(t, s) \in Z$ and $m$ is integrable on $[c, T]$ for each $c, 0<c \leq T$. Moreover, assume that the identically zero function is the only absolutely continuous function on $[0, c]$ which satisfies $\dot{u}(t)=h(t, u(t))$ a.e. on $[0, c]$ and for which the right derivative $D_{+} u(0)$ of $u(t)$ at $t=0$ exists and is 0 .

THEOREM 3.3. If we replace in the setting of Theorem 3.2 a Kamke function $w$ by a function $h$ and we suppose that $f^{d}$ is bounded and continuous, then problem (Q) has a solution. 
We omit the proof since it runs as in the proof of Theorem 3.2 except that we replace the use of Theorem 2.2 by that of Theorem 2.3 to find a continuous function $y_{1}$ such that $y_{1}=\xi$ on $[-d, 0]$ and for each $t \in\left[0, \frac{T}{n}\right]$

$$
y_{1}(t)=G(t, 0) \xi(0)+\int_{0}^{t} G(t, s) f_{1}\left(s, y_{1}(s)\right) d s .
$$

In fact, if $L(t) \neq 0$ our results generalize that of Gomaa [10] and Cichon [4], since we have a generalization of the compactness assumptions and in [4] the results are stated without delay. For the important case $L(t)=0$ we have, as a special case, a generalization of the existence theorems of Gomaa [13], Ibrahim-Gomaa [15], Papageorgiou [23], Cramer-Lakshmikantham-Mitchell [7], Szep [25] and Boundourides [2] in all of which the results are stated without delay. Szep in [25] studied the special case of problem (P) in a reflexive Banach space, Boundourides [2] and Cramer-Lakshmikantham-Mitchell [7] studied the special case of problem (P) in a nonreflexive Banach space, Papageorgiou [23] found weak solutions for the special case of problem (P) on a finite interval $I$ with $0<T<\infty$, Ibrahim-Gomaa [15] found weak solutions for the special case of problem $(\mathrm{P})$ on a finite interval $I$ and in [13] we give a generalization to recent results on the Cauchy problem by using weak and strong measures of noncompactness. Moreover in [11], [12] we study the nonlinear differential equations with and without delay while in [9] we study the differential inclusions with moving constraints.

ACKNOWLEDGMENT The author is very grateful to the editor and the referee for careful reading and for their valuable suggestions.

\section{REFERENCES}

1. Banas, J., and Goebel, K., Measure of noncompactness in Banach spaces, Lecture notes pure appl. math. 60, Marcel Dekker, New York 1980.

2. Boudourides, M., An existence theorem for ordinary differential equations in Banach spaces, Bull. Austral. Math. Soc. 22 (1980), 457-463.

3. Cichon, M., Differential inclusions and abstract control problems, Bull. Austral Math. Soc. 53 (1996), 109-122.

4. Cichon, M., On bounded weak solutions of a nonlinear differential equation in Banach space, Funct. Approx. Comment. Math. 21 (1992), 27-35.

5. Cichon, M., On measures of weak noncompactness, Publ. Math. Debrecen 45 (1994), 93-102.

6. Cichon, M., Trichotomy and bounded solutions of nonlinear differential equations, Math. Bohem. 119 (1994), 275-284.

7. Cramer, E., Lakshmikantham, V., and Mitchell, A. R., On the existence of weak solutions of differential equations in nonreflexive Banach spaces, Nonlinear Anal. 2 (1978), 169-177.

8. Deimling, K., Ordinary Differential Equations in Banach Spaces, Lecture Notes in Math. 596, Springer, Berlin 1977. 
9. Gomaa, A. M., Existence of solutions for differential inclusions on closed moving constraints in Banach spaces, Electron. J. Differential Equations 22 (2009), 10 pp.

10. Gomaa, A. M., Existence solutions for differential equations with delay in Banach spaces, Proc. Math. Phys. Soc. Egypt 84 (2006), 1-12.

11. Gomaa, A. M., On bounded weak and pseudo-solutions of nonlinear differential equations having trichotomy with and without delay in Banach spaces, Int. J. Geom. Methods Mod. Phys. 7 (2010), 357-366.

12. Gomaa, A. M., On theorem for weak solutions of nonlinear differential equations with and without delay in Banach Spaces, Comment. Math. Prace Mat. 47 (2007), 179-191.

13. Gomaa, A. M., Weak and strong solutions for differential equations in Banach spaces, Chaos Solitons Fractals 18 (2003), 687-692.

14. Hille, E., and Phillips, R. G., Functional analysis and semi-groups, rev. ed. Colloq. Publ. 31, Amer. Math. Soc., Providence, RI 1957.

15. Ibrahim, A. G., and Gomaa, A. M., Strong and weak solutions for differential inclusions with moving constraints in Banach spaces, Pure Math. Appl. 8 (1997), 53-65.

16. Krzyska, S., and Kubiaczyk, I., On bounded pseudo and weak solutions of a nonlinear differential equation in Banach spaces, Demonstratio Math. 32 (1999), 323-330.

17. Kubiaczyk, I., On the existence of solutions of differential equations in Banach spaces, Bull. Polish Acad. Sci. Math. 33 (1985), 607-614.

18. Kubiaczyka, R., Zastosowanic miar niezwartosći w teorii róńrózniczkowych i mathcalkowych, Oh D, thesis, Lublin 1978.

19. Makowiak, M., On some bounded solutions of a nonlinear differential equations, Demonstratio Math. 30 (1997), 801-808.

20. Massera, J. L., and Shaffer, J. J., Linear differential equations and function spaces, Pure and Appl. Math. 21, Academic Press, New York 1966.

21. Mitchell, A. R., and Smith, C., An existence theorem for weak solutions of differential equations in Banach spaces, pp. 387-403 in: Nonlinear equations in abstract spaces, Proc. Int. Symp., Arlington 1977, Academic Press, New York 1978.

22. Olech, O., On the existence and uniqueness of solutions of an ordinary differential equation in the case of Banach space, Bull. Acad. Polon. Sci. 8 (1960), 667-673.

23. Papageorgiou, N. S., Weak solutions of differential equations in Banach spaces, Bull. Austral. Math. Soc. 33 (1986), 407-418.

24. Pazy, A., Semigroups of linear operators and applications to partial differential equations, Appl. Math. Sci. 44, Springer, Berlin 1983.

25. Szep, A., Existence theorem for weak solutions of ordinary differential equations in reflexive Banach spaces, Studia Sci. Math. Hungar. 6 (1971), 197-203.

26. Szufla, S., On the existence of solutions of differential equations in Banach spaces, Bull. Acad. Polon. Sci. 30 (1982), 507-515.

\author{
MATHEMATICS DEPARTMENT \\ FACULTY OF SCIENCE \\ HELWAN UNIVERSITY \\ CAIRO \\ EGYPT \\ E-mail: gomaa_5@hotmail.com
}

